\title{
Patrones comportamentales de fóridos (Diptera: Phoridae) parasitoides de Atta colombica (Hymenoptera: Formicidae)
}

Robin Bedoya Cochet, ${ }^{1}$ Ángela Ortega León ${ }^{2}$ \& Adriana Ortiz-Reyes*3

1. Universidad de Córdoba, Facultad de Ciencias Básicas, Departamento de Biología, Carrera 6 No. 76-103 Montería, Colombia; robindavidbc28@gmail.com

2. Grupo de Biodiversidad, Universidad de Córdoba, Facultad de Ciencias Básicas, Departamento de Biología, Carrera 6 No.76-103 Montería, Colombia; amortega25@yahoo.com.mx

3. Grupo de Sustancias Activas y Biotecnología, Universidad Nacional de Colombia, sede Medellín, Facultad de Ciencias, Calle 59A No 63 - 20 Medellín, Colombia; adortizr@unal.edu.co

* Correspondencia

Recibido 01-VI-2016. Corregido 18-I-2017. Aceptado 13-II-2017.

\begin{abstract}
Behavioral patterns of parasitoid phorids (Diptera: Phoridae) of Atta colombica (Hymenoptera: Formicidae). In ecology, the success of parasitoid-host interactions is determined by the behavior of seeking, recognition, attack and defense deployed by the participants in the interaction. Our study aimed to understand the behavioral patterns between parasitoid phorids and their host Atta colombica from a forest fragment of Córdoba department, Colombia. We observed three nests of Atta colombica, from December 2013 to Januray 2015 (including dry and rainy seasons), for a total effort of 189 hours. We observed Phorids, their attacks to ants and collected their loads; we also considered differences in the way the parasitoids attacked and observed the microhabitat they preferred. A total of 52 individuals of Eibesfeldtphora attae and 54 of Apocephalus colombicus were collected. Apparently there was no preference for the size of the ants that were attacked by phorids, but larger loads were selected to land on them. When the ants responded to the phorid attack, they used individual defenses (adopting positions or increasing their speed) or grupal defenses (closer ants attacking the phorid). Parasitoid phorids have specialized their behavior, in order to ensure a successful attack on their host, who responded by modifying its behavior, according to the attacker (parasitoid). Rev. Biol. Trop. 65 (2): 461473. Epub 2017 June 01.
\end{abstract}

Key words: leaf-cutting ants, host behavior, host selection, interactions, parasitism.

El estudio de las interacciones ecológicas en diversos grupos taxonómicos ha consolidado un marco teórico sólido y muy completo que aborda las interacciones parasitoide-hospedero (Santos \& Quicke, 2011). Sin embargo, algunos patrones y aspectos teóricos no se pueden generalizar para todas las interacciones parasitoide-hospedero, debido a las diferencias en el origen filogenético de los parasitoides, los tipos de señales usadas para la selección de hospederos, las interacciones ecológicas y la distribución geográfica de los participantes en la interacción (Elizalde, 2009; Elizalde \& Folgarait, 2010; Mathis \& Philpott, 2011).
Algunas especies de dípteros de la familia Phoridae están entre los enemigos naturales de las hormigas cortadoras de hojas, porque son sus parasitoides (Feener \& Moss, 1990; Brown, 1997; Elizalde \& Folgarait, 2010). Estos dípteros deben superar una serie de barreras físicas y de comportamiento que imponen sus hospederos para poder alcanzar el éxito reproductivo (Feener \& Brown, 1997; Elizalde \& Folgarait, 2011); características que son cruciales en el entendimiento de las interacciones a nivel ecológico (Elizalde, 2009). De estas moscas parasitoides se conoce que afectan varias especies de hormigas de los géneros Atta y Acromyrmex, 
pero poco se sabe sobre la biología y ecología de estas interacciones (Brown, 1997), por lo cual se desconoce qué tan significativa es la importancia de estos parasitoides.

Para el género Atta se han descrito 39 especies de parasitoides ubicados en cinco géneros. Los más importantes, por su variedad de hospederos y potencialidad en el control biológico, y de mayor distribución geográfica son Apocephalus Coquillett, Eibesfeldtphora Disney y Myrmosicarius Borgmeier (Elizalde, 2009; Folgarait, 2013). Entre los diferentes efectos que pueden provocar sobre las hormigas cortadoras de hoja, se encuentra la disminución del reclutamiento hacia las fuentes de forrajeo, disminución en el tamaño de los fragmentos transportados hacia la colonia, pérdida de interacciones competitivas por la comida e, incluso, el abandono de las fuentes de alimento (Feener \& Moss, 1990; Folgarait, 2013). También, se puede estimular el despliegue de posturas defensivas de protección con el cuerpo y la asociación con pequeñas hormigas compañeras sobre las cargas que impiden el ataque de los fóridos (Feener \& Moss 1990, Elizalde, 2009; Elizalde \& Folgarait, 2011).

Dado que cada especie de fórido parasitoide utiliza diferentes señales para detectar sus hospederos, y despliega un comportamiento de oviposición particular, es necesario estudiar los patrones de comportamiento de cada especie parasitoide (Bragança, Tonhasca, \& Moreira, 2002; Mathis \& Philpott, 2011); así como las respuestas defensivas de las hormigas ante los ataques, porque de esto depende la formación y mantenimiento de sus interacciones (Fellowes \& Godfray, 2000; Elizalde, 2009). Los comportamientos descritos tienen una base genética, se estructuraron y optimizaron por procesos de selección natural (Roitberg, Boivin, \& Vet, 2001; Rodríguez \& Arredondo-Bernal, 2007) y por mantenerse a lo largo del tiempo, permiten dilucidar el efecto sobre el moldeado evolutivo de estas interacciones ecológicas (Elizalde, 2009) y apoyar en la comprensión de la comunicación multimodal entre dichas especies (Mathis \& Philpott, 2011).
El objetivo de este trabajo fue describir las estrategias de ataque empleadas por los parasitoides y la respuesta desplegadas por las hormigas de la especie $A$. colombica ante estos ataques. Adicionalmente, se evaluó la preferencia de los parasitoides por los microhábitats de los nidos, el tamaño de las hormigas atacadas, el efecto del tamaño y forma de la carga de la hormiga en el ataque y, la influencia de la época del año y la actividad de las hormigas en la abundancia de los fóridos.

\section{MATERIALES Y MÉTODOS}

Área de estudio: El estudio se realizó en un fragmento de bosque seco tropical (Bs-T) de 10 hectáreas aproximadamente, ubicado en la finca Santa Isabel, en el kilometro 26 de la vía Montería-Planeta Rica, departamento de Córdoba, Colombia.

El departamento de Córdoba se encuentra ubicado aproximadamente a $20 \mathrm{msnm}$. Su temperatura promedio es de $28^{\circ} \mathrm{C}$, se localiza entre los $09^{\circ} 26^{\prime} 16^{\prime \prime} \mathrm{N}-74^{\circ} 47^{\prime} 43^{\prime \prime} \mathrm{W} \& 0^{\circ} 22^{\prime} 05^{\prime}$ " N - 76³0'01" W. Según la clasificación climatológica de Holdridge (1987) presenta tres tipos de zonas de vida: bosque seco tropical, bosque húmedo tropical y transición entre bosque seco y bosque húmedo tropical. Cuenta con una precipitación media anual de $1200 \mathrm{~mm}$, con época lluviosa (mayo-noviembre) y época seca bien marcadas (diciembre-abril) (Palencia, Mercado, \& Combatt, 2006).

El trabajo de campo se llevó a cabo en diciembre 2013 y enero 2015. Inicialmente, se seleccionaron tres nidos maduros y activos, separados entre sí por $200 \mathrm{~m}$, ubicados en el interior, borde y exterior del fragmento de bosque (Cuadro 1). En cada nido se definieron cuatro microhábitats: boca activa de entrada al nido (BOAC); el camino de forrajeo (CAMF), la boca o salida al basurero (SABA) y el basurero externo o montaña de detrito (DET). Se realizaron un total de 21 muestreos, en el área de estudio.

Fóridos parasitoides de Atta colombi$c a$ : En cada nido se realizaron observaciones 
CUADRO 1

Características, actividad promedio y localización de los nidos de Atta colombica en un fragmento de bosque

TABLE 1

Characteristics, average activity and location of the nests of Atta colombica in the forest fragment

\begin{tabular}{cccccccc} 
Nido\# & Ubicación & Área $\left(\mathrm{m}^{2}\right)$ & $\mathrm{Nb}$ & $\mathrm{Nc}$ & Activ & $\mathrm{N}$ & W \\
1 & Interior & 129.95 & 59 & 169 & 177.77 & $08^{\circ} 34^{\prime} 22.1^{\prime \prime}$ & $075^{\circ} 42^{\prime} 00.9^{\prime \prime}$ \\
2 & Borde & 155.76 & 46 & 42 & 75.44 & $08^{\circ} 34^{\prime} 23.8^{\prime \prime}$ & $075^{\circ} 41^{\prime} 55.6^{\prime \prime}$ \\
3 & Exterior & 66.12 & 67 & 94 & 179.24 & $08^{\circ} 34^{\prime} 29.9^{\prime}{ }^{\prime}$ & $075^{\circ} 42^{\prime} \mathrm{O} 3.4^{\prime \prime}$ \\
\hline
\end{tabular}

$\mathrm{Nb}$ = número de bocas por nido, Ubicación = ubicación de los nidos en el fragmento, $\mathrm{Nc}=$ número de caminos activos en todos los muestreos, Activ = actividad promedio de hormigas (hormigas/minuto).

$\mathrm{Nb}=$ number of mouths per nest, Location $=$ location of nests in the fragment, $\mathrm{Nc}=$ number of active paths in all samples, Activ $=$ average ants activity (ants/minute).

de las hormigas y los fóridos, evitando la menor perturbación posible. Se capturaron fóridos presentes en los cuatro microhábitats de cada nido con ayuda de un aspirador entomológico. Las muestras se depositaron en tubos de microcentrífuga tipo ependorff con alcohol al $70 \%$, luego fueron procesadas e identificadas en el Laboratorio de Biodiversidad de la Universidad de Córdoba, con ayuda de estereoscopio y microscopio (Leica). La identificación se hizo con base en claves para géneros (Brown et al., 2010) y especies (Brown, 1997; Uribe, Brown, Bragança, Queiroz, \& Nogueira, 2014). Las muestras biológicas se depositaron en las Colecciones Zoológicas de la Universidad de Córdoba con un duplicado en el Museo Entomológico Francisco Luis Gallego de la Universidad Nacional de Colombia (sede Medellín) con número de catálogo 1CM011-1CM169, 2CM014-2CM170 y 3CM025-3CM165.

Evaluación del comportamiento de los fóridos parasitoides: El comportamiento de los fóridos se describió mediante observaciones en campo, por un periodo de dos horas y media (observaciones de 30 minutos en cada microhábitat con un intervalo de 10 minutos entre microhábitat), iniciando las observaciones después de verificar la actividad del nido (flujo continuo de hormigas con carga) hasta revisar los tres nidos entre las 06:00 y 18:00 h; cada nido fue revisado 31 veces. El orden del horario de las observaciones de los nidos, fue sorteado previamente para evitar el efecto de la hora de lectura.

Una vez que se detectó el fórido, se inició la descripción del comportamiento durante el acecho y ataque sobre $A$. colombica, las conductas de las moscas se clasificaron según las categorías definidas por Elizalde (2009): (i) posado, cuando el fórido se ubicó sobre el costado del camino o donde se encontraban las hormigas; (ii) posado sobre hoja, cuando el fórido se encontró sobre el fragmento de hoja transportada por la hormiga; (iii) vuelo de inspección, cuando se observó a la mosca volando sin dirección aparentemente; (iv) vuelo de acecho, si estaba rondando a unos $5 \mathrm{~cm}$ por encima de los hospederos; (v) intento de ataque, cuando las moscas levemente tocaron las hormigas; y (vi) ataque, cuando hubo penetración aparente del ovipositor.

Evaluación del comportamiento de las hormigas ante la presencia y ataque de fóridos: La respuesta de las hormigas contra los ataques de los fóridos se describió igualmente durante los avistamientos, clasificando los comportamientos en categorías de acuerdo con Elizalde y Folgarait (2011): (i) ninguna, cuando las hormigas no tuvieron ninguna reacción; (ii) aumento de velocidad, cuando evidentemente la hormiga aumentó de velocidad aunque no se midiera; (iii) persuasión, cuando la hormiga 
trató de retirar la mosca con sus patas y antenas; (iv) adopción de alguna postura, cuando la hormiga adoptó una postura especial para evitar el ataque del fórido; y (v) ataque directo, cuando la hormiga atacó directamente al fórido.

Durante la observación directa en campo, se tomaron fotografías y videos de los despliegues de comportamiento de los fóridos y las respuestas de las hormigas para describir detalladamente las conductas y la duración de cada comportamiento.

Variables relacionadas con la presencia de fóridos: Para estudiar la similitud entre los nidos y medir el efecto de la disponibilidad de hormigas, se utilizaron los valores promedio del ancho y largo del nido para calcular el área de cada nido, y tomarla como medida indirecta del tamaño de la colonia, el promedio del número de caminos de forrajeo durante todo el estudio y la actividad promedio. Para esta última, antes y después de cada avistamiento en cada zona del nido, se registró la actividad de las hormigas contando el número de hormigas con carga que pasaban por un punto durante un minuto, con ayuda de videos grabados antes y después de los 30 min de observación.

Se registró la hora exacta de cada observación de los fóridos para relacionar las horas de mayor actividad. Con el objetivo de realizar comparaciones, las hormigas atacadas por los fóridos se capturaron y llevaron al laboratorio, donde se midió el tamaño de estas y compararon con una muestra de hormigas al azar que también fueron recolectadas y medidas. Las cargas de las hormigas sobre las cuales se posaron los fóridos y otra muestra al azar, también se recolectaron y midieron para compararlas. Las mediciones se realizaron con la ayuda del software Image Tool 3.0 (IT3). Se registró la forma de la carga de la hormiga, teniendo en cuenta si era un fragmento de hoja aplanada (comprimida bilateralmente) u alguna semilla o botón floral en forma cilíndrica, con el fin de detectar si había alguna preferencia.

Para el análisis de los datos se emplearon los paquetes estadísticos Statgraphics centurion XVI y XLSTAT versión 2009. Se aplicaron pruebas de Shapiro-wilk con el fin de determinar si los datos seguían una distribución normal. Se aplicó un ANOVA para ver si existían diferencias significativas entre los nidos (abundancia de fóridos por nido), y con una prueba de Chi-cuadrado se determinó si el microhábitat influyó en la presencia del parasitoide. Con los datos de fragmentos de hojas recolectados, sobre los cuales se posaron los fóridos y una muestra al azar de fragmentos de cada nido, se emplearon pruebas de Kruskal-Wallis para determinar si el tamaño de las cargas afectaba el aterrizaje de las moscas sobre los fragmentos. Mediante una prueba U de Mann-Whitney (Wilcoxon) y una t-student se compararon las medidas de las hormigas atacadas con una muestra al azar de hormigas que no lo fueron. Con el fin de evaluar el efecto del tiempo, la actividad promedio del nido y el número de caminos activos sobre la abundancia de fóridos, se ajustó un modelo lineal generalizado (GLM). Finalmente, se construyó un etograma y se compararon las frecuencias de aparición de las categorías de comportamiento.

\section{RESULTADOS}

Fóridos presentes en los nidos de $\mathrm{Atta}$ colombica: Se efectuaron $186 \mathrm{hr}$ de observación, en las que se capturaron manualmente un total de 106 fóridos parasitoides de $A$. colombi$c a$, perteneciente a dos especies: 54 individuos de Apocephalus colombicus Brown, 1997, y 52 de Eibesfeldtphora attae Disney, 1996.

Características de los nidos y abundancia de fóridos: No hubo una relación directa de la actividad de los nidos con la abundancia de las dos especies de fóridos (E. attae, $\mathrm{r}^{2}=0.072$, $\mathrm{F}=1.554, \mathrm{gl}=1, \mathrm{P}=0.227$ y A. colombicus, $\mathrm{r}^{2}=0.047, \mathrm{~F}=1.445, \mathrm{gl}=1, \mathrm{P}=0.239$ ). Por otra parte, la abundancia de fóridos no se vio influenciada por las variables (hora de colecta, actividad de hormigas y número de caminos activos) empleadas para el modelo lineal generalizado $(\mathrm{F}=0.79, \mathrm{gl}=14, \mathrm{P}=0.670)$. 
Distribución por nidos: No hubo una diferencia significativa para la abundancia de parasitoides entre los nidos muestreados $(\mathrm{F}=$ 7.25, $\mathrm{gl}=2, \mathrm{P}=0.070)$. Las dos especies de fóridos estuvieron presentes en los tres nidos (Fig. 1).

Preferencia de los fóridos por los microhábitats del nido: La presencia de los parasitoides dependió del microhábitat $\left(\mathrm{X}^{2}=52.389\right.$, $\mathrm{gl}=3, \mathrm{P}<0.01)$. En los cuatro microhábitats se registraron fóridos, siendo el camino de forrajeo y la salida al basurero los que mostraron la mayor abundancia (46 y $24 \%$, respectivamente), seguido de las bocas activas y del detrito (21 y $9 \%$ respectivamente) (Fig. 1).

A nivel de especie, para la búsqueda de hospederos, A. colombicus prefirió el camino de forrajeo en su mayoría ( $80 \%$ ), mientras $E$. attae concentró su búsqueda en la salida al basurero (40\%). De igual manera, el ataque y los intentos de acceso de los dos parasitoides fueron centrados en estos mismos sitios, $A$. colombicus en el camino de forrajeo $(88.46 \%)$ y E. attae en la salida al basurero (51.61\%).

Fluctuación anual y horarios de actividad: A. colombicus y E. attae presentaron horarios de actividad amplios y con aspectos similares. Se observaron fóridos en las diferentes categorías de comportamiento desde la 07:10 hasta las 17:40 y los ataques e intentos de ataques se registraron desde las 07:40 hasta las 17:00. La presencia de A. colombicus mostró una relación curvilínea con la hora de muestreo, ajustándose los datos con una significancia del $65.9 \%$ a una regresión polinómica $(\mathrm{F}=9.66, \mathrm{gl}=2, \mathrm{P}=0.004)$, indicando las mayores probabilidades de encontrar este fórido en la escala temporal desde las 10:00 a las 15:00 (Fig. 2). Eibesfeldphora attae mostró un comportamiento con mayor plasticidad, distribuyéndose a diferentes horarios a lo largo del día (Fig. 2). El parasitoide A. colombicus estuvo presente en casi todos los muestreos. La relación entre la abundancia y la época del año

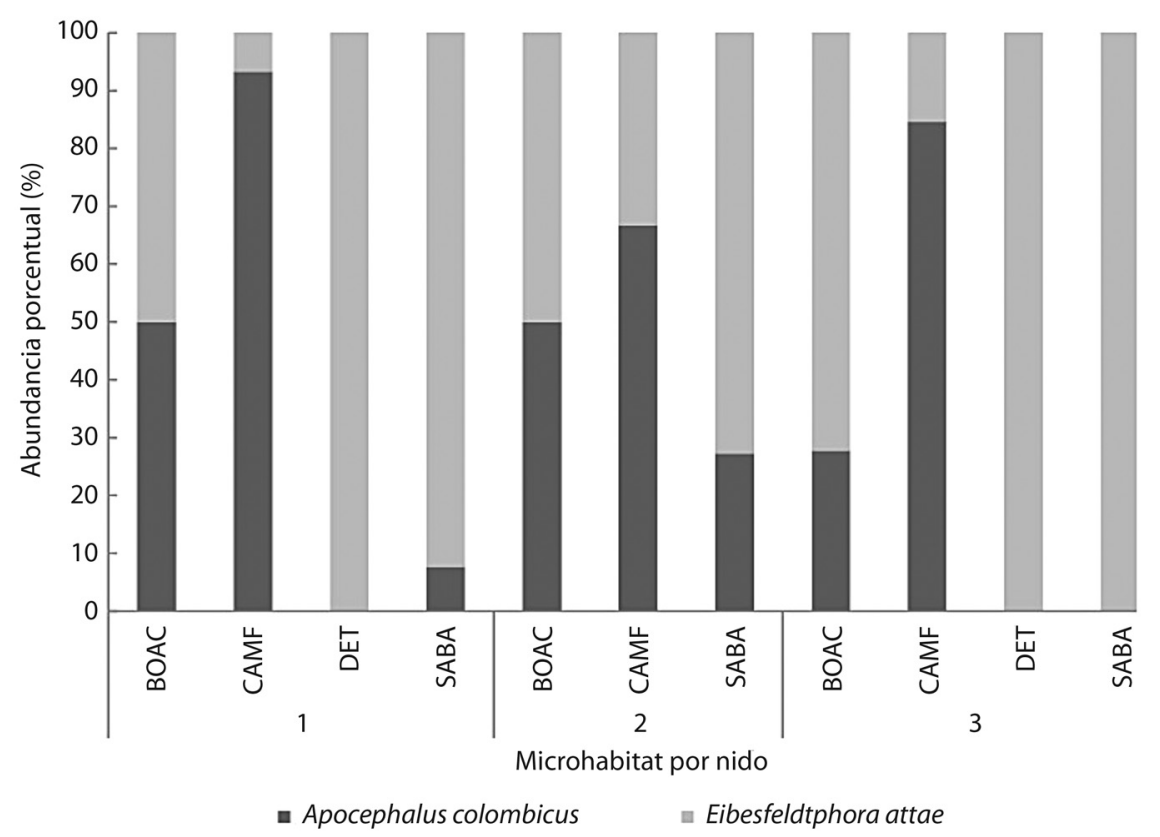

Fig. 1. Abundancia porcentual de fóridos por nido y microhábitat. $\mathrm{SABA}=$ salida al basurero, $\mathrm{DET}=$ detrito, $\mathrm{CAMF}=$ caminos de forrajeo, BOAC $=$ bocas activas.

Fig. 1. Abundance percentage of phorids per nest and microhabitat. $\mathrm{SABA}=$ output to the dump, $\mathrm{DE}=$ detritus, $\mathrm{CAMF}=$ foraging paths, $\mathrm{BOAC}=$ active mouths. 


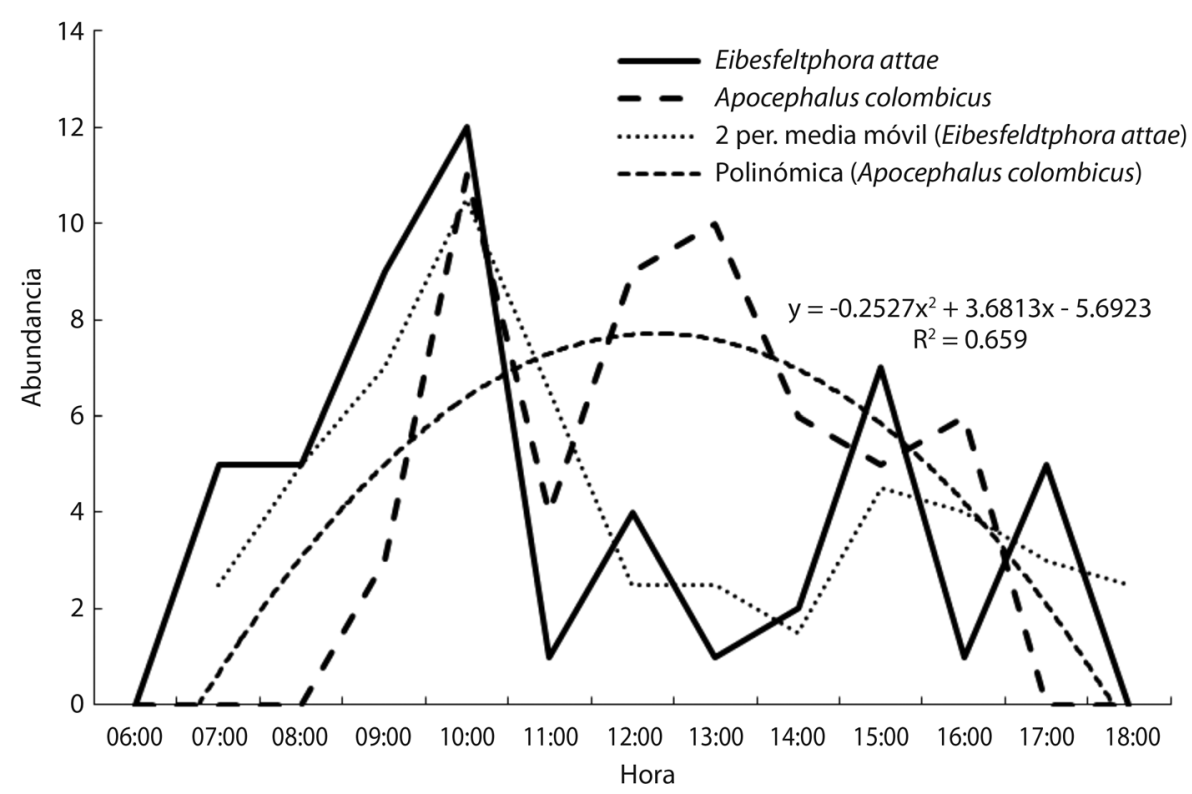

Fig. 2. Curva estimada del efecto de la hora de muestreo sobre la abundancia de Apocephalus colombicus y Eibesfeldtphora attae.

Fig. 2. Estimated curve of the effect of sample time on the abundance of Apocephalus colombicus and Eibesfeldtphora attae.

no mostró una tendencia clara, aunque podemos indicar los picos más altos desde febrero hasta marzo del 2014 y desde septiembre del mismo año hasta enero del siguiente año (Fig. 3). La abundancia de $E$. attae fue mayor en la época seca, ajustándose los datos a una regresión polinómica $(\mathrm{F}=5.96, \mathrm{gl}=2, \mathrm{P}=0.019)$; este fórido se presenta desde noviembre hasta marzo y desaparece casi completamente en los demás meses (Fig. 3).

Sitio de oviposición: Las dos especies de fóridos difieren en el lugar de oviposición, $A$. colombicus siempre atacó a las hormigas en el área mandibular, mientras que E. attae se observó atacando a hormigas en el gáster y en el mesosoma (63.63 y $36.36 \%$, respectivamente).

Efecto del tamaño y la forma de las cargas: El análisis individual del tamaño de las cargas no mostró diferencias significativas, por lo tanto, se unificaron los datos para tener un análisis más robusto. Se encontró una diferencia significativa en cuanto al tamaño de la carga de la hormiga utilizada por los parasitoides para posarse sobre ella $\left(\mathrm{X}^{2}=17.2716\right.$, $\mathrm{P}$ $<0.01$ ), prefiriendo hormigas con cargas de un tamaño mayor $\left[141.46 \mathrm{~mm}^{2}(\mathrm{CV}=37.73 \%)\right]$ al promedio normal [98.18 $\mathrm{mm}^{2}(\mathrm{CV}=38.52 \%$ ]. Por otra parte, de las 43 cargas registradas para A. colombicus, el $67.44 \%$ fueron fragmentos de hojas o material vegetal con formas aplanadas (comprimidas bilateralmente), el $32.56 \%$ restante fueron fragmentos de tallos, botones florales y material vegetal con forma circular o cilíndrica.

Efecto del tamaño de las hormigas: No hubo una preferencia por el tamaño de las hormigas atacadas por A. colombicus (W de MannWhitney, $\mathrm{W}=6.0$, valor $-\mathrm{p}=0.946$ ) (Fig. 4). De igual manera no se encontró preferencia de $E$. attae por hormigas de un tamaño mayor (t-student, $\mathrm{t}=-0.0590$, valor- $\mathrm{p}=0.9533$ ) (Fig. 4).

Comportamiento de $A$. colombicus: Inicia su despliegue desde una percha sobre la vegetación de porte bajo, cerca de los caminos 


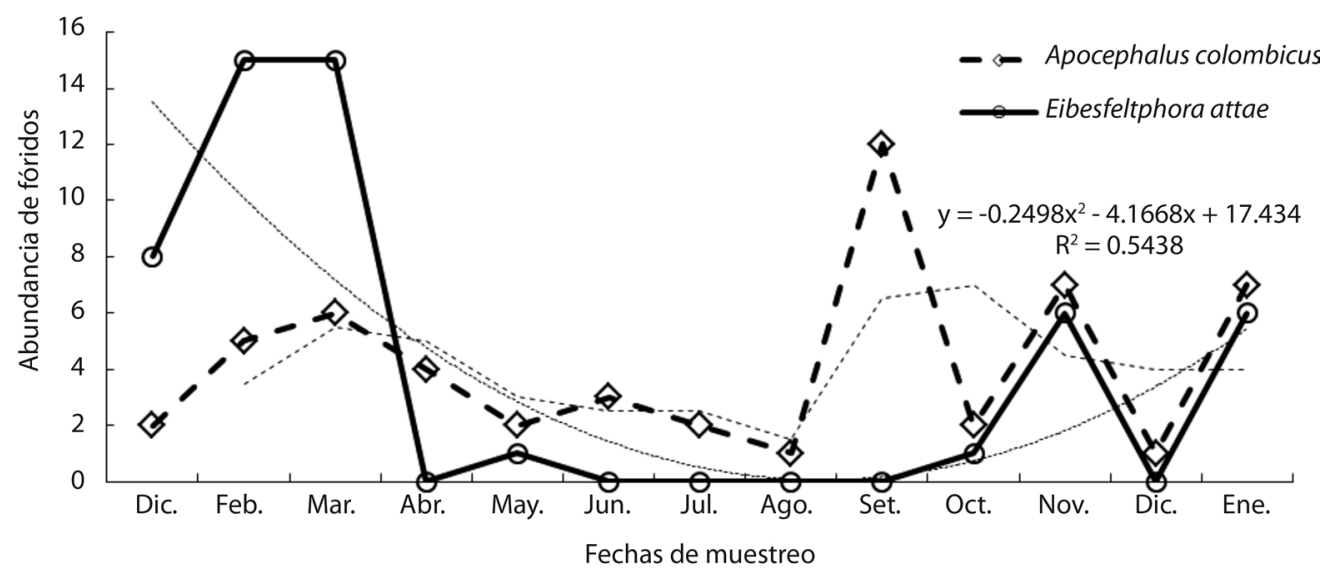

Fig. 3. Curva estimada del efecto de los meses de muestreo sobre la abundancia de Apocephalus colombicus y Eibesfeldtphora attae.

Fig. 3. Estimated curve of the effect of sample months on the abundance of Apocephalus colombicus and Eibesfeldtphora attae.

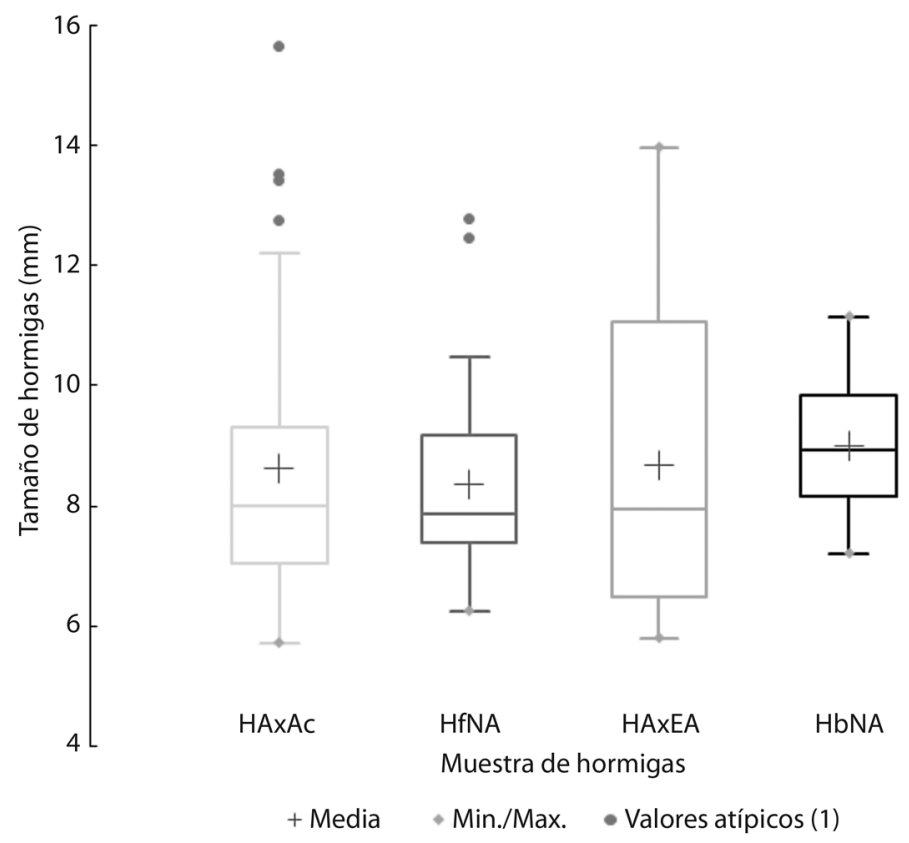

Fig. 4. Comparación del tamaño de las hormigas atacadas con una muestra al azar de hormigas no atacadas. HAxAc = hormigas atacadas por $A$. colombicus, HfNA = hormigas no atacadas por $A$. colombicus, HAxEa = hormigas atacadas por $E$. attae y $\mathrm{HbNA}=$ hormigas no atacadas por $E$. attae.

Fig. 4. Comparison of the size of attacked ants with a random sample of not attacked ants. HAxAc $=$ attacked ants by $A$. colombicus, HfNA = not attacked ants by . colombicus, $\mathrm{HAxEa}=$ attacked ants by $E$. attae and HbNA = not attacked ants by E. attae. 
o en la hojarasca y ramas secas circundantes a las hormigas por $146.66 \pm 185.02$ s. Continúa con un vuelo de búsqueda sobre las hormigas que llevan cargas por $17.18 \pm 34.42 \mathrm{~s}$, o algunas veces pasa directamente de su percha a los fragmentos de hojas que cargan las hormigas aterrizando en la punta de la carga donde se posa por $5.66 \pm 3.03 \mathrm{~s}$. Luego se desplazan hacia la base donde las mandíbulas sostienen el fragmento, e introduce su ovipositor al interior de las mandíbulas tratando de ovipositar sobre las hormigas por más de $6.5 \pm 5.64 \mathrm{~s}$ (Fig. 5). Apocephalus colombicus no le da oportunidad de defenderse a la hormiga, es una especie que aprovecha la tarea que realiza la hormiga mientras lleva su carga para concretar su ataque.

Comportamiento de E. attae: Este fórido se posa por $180.33 \pm 300.51$ s sobre la hojarasca, ramas secas o directamente sobre el suelo y cerca de las hormigas (Fig. 5). Continúa con un vuelo de búsqueda sobre las hormigas a una distancia aproximada de $10 \mathrm{~cm}$ por $42.0 \pm$ $28.90 \mathrm{~s}$ y cuando aparentemente selecciona una hormiga, la acecha volando por detrás a menos de $5 \mathrm{~cm}$ de la hormiga por $32.411 \pm 31.32 \mathrm{~s}$, y ataca a la hormiga en el gáster o en el mesosoma con movimientos belicosos y muy rápidos sin ningún aterrizaje necesario en sólo $1.57 \pm$ $0.78 \mathrm{~s}$. Este fórido debe acercarse demasiado a las hormigas durante el acecho, por lo cual puede darle la oportunidad a la hormiga de ser capturado como respuesta de defensa.

Comportamiento de las hormigas ante la presencia y ataque de fóridos: $\mathrm{El} 79.1 \%$ de las hormigas no mostraron una respuesta ante el ataque de los parasitoides, mientras el 20.9 $\%$ respondió al ataque de los parasitoides y el despliegue de la conducta dependió de la especie que atacaba. Se presentaron dos diferentes tipos de respuesta, la grupal y la individual, que se detallan a continuación.

La respuesta grupal se generó cuando $E$. attae intentaba atacar a una hormiga y una o varias hormigas vecinas trataban de atacar al fórido levantando sus mandíbulas hacia el parasitoide, para impedir su ataque. Se registró el caso en que varias hormigas vecinas atraparon al fórido y lo atacaron cortándolo con sus mandíbulas (4.47\%) (Fig. 6F). Otra repuesta grupal se dio cuando A. colombicus se posaba sobre el fragmento vegetal y aparentemente era reconocido por la hormiga obrera, la cual detenía la marcha, bajaba su carga y una hormiga de menor tamaño subía a la hoja o material vegetal que estuviera transportando, para impedir que el parasitoide volviera a atacar (1.49\%); comportamiento registrado en otros estudios denominado "autostop" o polizón, que además de otras funciones puede evitar

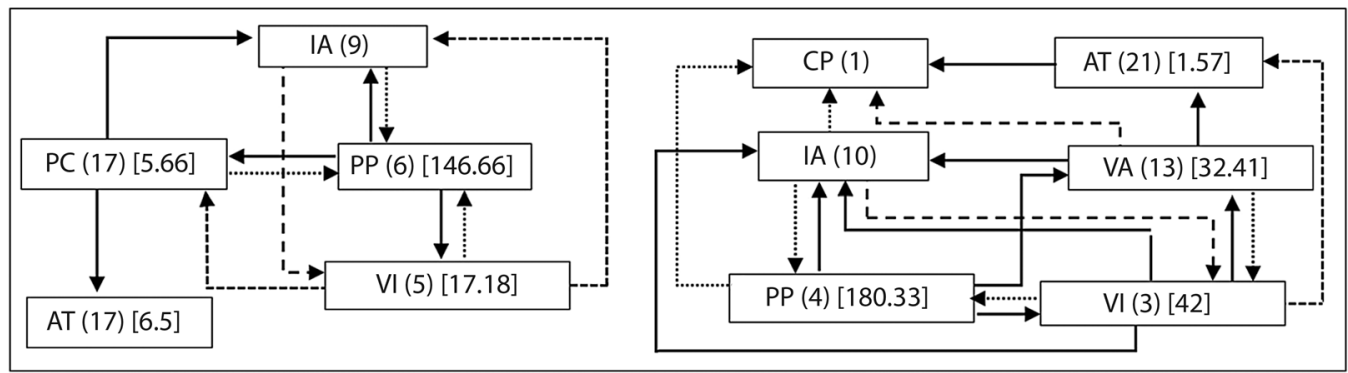

Fig. 5. Izquierda, etograma de E. attae; derecha, etograma de A. colombicus. Abreviaturas: $\mathrm{CP}=$ capturado por las hormigas; $\mathrm{IA}=$ intento de ataque; $\mathrm{PP}=$ posado en percha; $\mathrm{AT}=$ ataque; $\mathrm{VI}=$ vuelo de inspección; $\mathrm{VA}=$ vuelo de acecho; $\mathrm{PC}=$ posado en la carga de la hormiga; ()$=$ frecuencia del comportamiento; [] = promedio del tiempo en segundos.

Fig. 5. Left, E. attae ethogram; right, A. colombicus ethogram. Abbreviations: $\mathrm{CP}=$ captured by ants; $\mathrm{IA}=$ attempted attack; $\mathrm{PP}=$ perched on hanger; $\mathrm{AT}=$ attack; $\mathrm{VI}=$ inspection flight; $\mathrm{VA}=$ stalking flight; $\mathrm{PC}=$ perched on the load ant; ()$=$ Frequency of the behavior; [] = Average of time in seconds. 

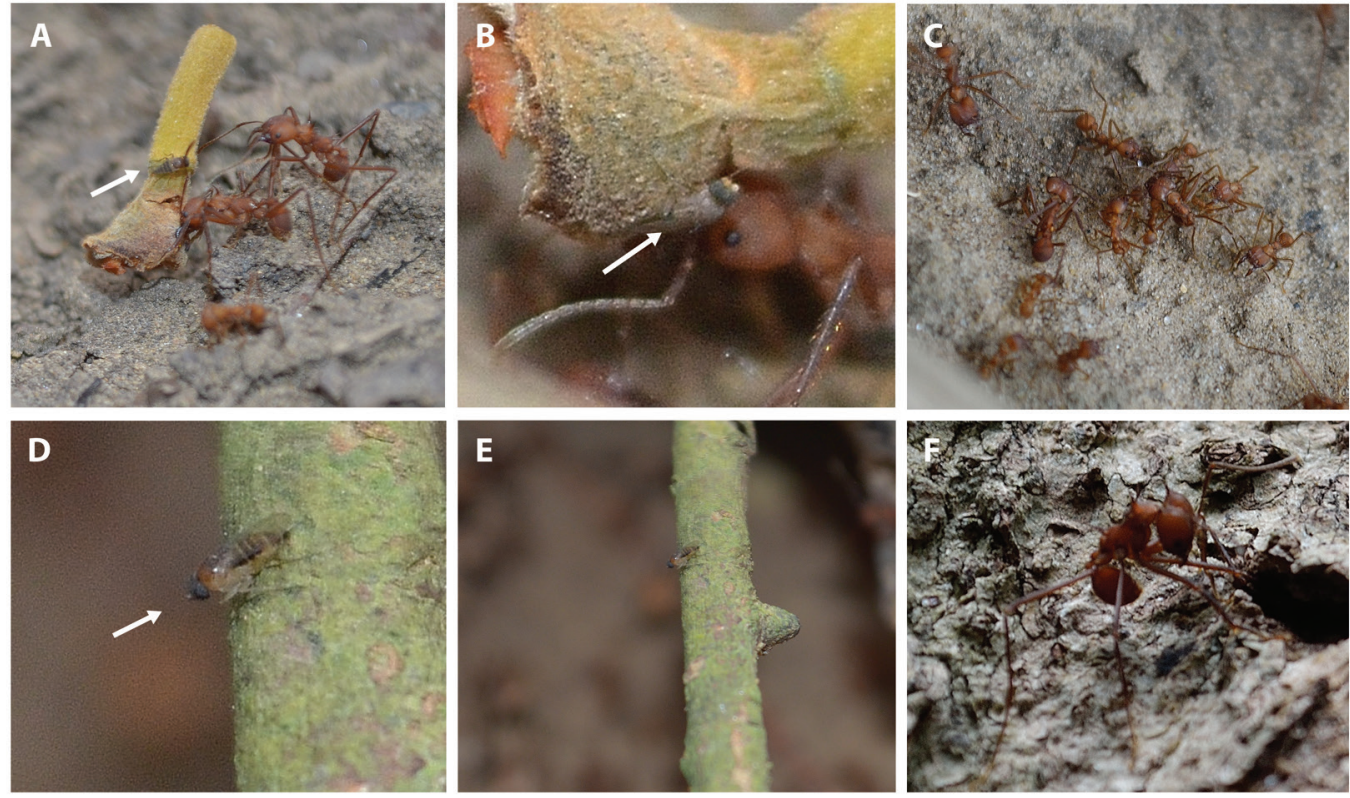

Fig. 6. Categorías conductuales de los fóridos Apocephalus colombicus (A y B, fórido atacando en el área mandibular) y Eibesfeldtphora attae (D y E, acechando hormigas del basurero) durante el ataque a las hormigas. $\mathbf{C}$ y $\mathbf{F}$, conducta de respuesta de las hormigas ante los ataques, posición en c y ataque grupal respectivamente.

Fig. 6. Behavioral categories of phorids Apocephalus colombicus (A and B, phorid attacking in the mandib area) and Eibesfeldtphora attae (D and $\mathbf{E}$, stalking ants from dump) during the attack of the ants. $\mathbf{C}$ and $\mathbf{F}$, response behavior of ants before attack, position in c and group attack respectively.

posibles ataques de estos parasitoides en esta misma especie de hormiga.

La respuesta individual de $A$. colombica se dio de diferentes maneras al ser atacada por E. attae. Cuando detectaban al fórido, algunas hormigas se dirigían directamente a él utilizando sus mandíbulas para atacarlo (1.49\%); otras detenían la marcha y apoyadas en las patas traseras, intentaban detener o persuadir a la mosca utilizando las patas delanteras (1.49\%); algunas hormigas optaron por colocar el gáster entre las patas y orientado hacia delante tomaban una posición en forma de "C" y avanzaban lentamente por el camino (2.98 \%) (Fig. 6C). Finalmente, el $5.97 \%$ de las hormigas, cuando se percataba de la cercanía de E. attae, generaba un claro aumento en la velocidad de desplazamiento hasta alejarse del fórido.

Cuando A. colombicus fue el atacante, una conducta reflejada por las hormigas fue soltar la carga, agachar la cabeza por debajo del tórax y bajar el gáster entre las patas (categoría "hacerse bolita", $1.49 \%)$. Por último, hubo hormigas que ante la presencia de $\mathrm{A}$. colombicus, detuvieron su marcha y se encogieron en posición boca abajo como pegando el cuerpo al suelo $(1.49 \%)$.

\section{DISCUSIÓN}

La abundancia de fóridos que se registró (106) en los 14 meses de muestreo es consistente con trabajos realizados en fóridos como Da Silva (2011), quien encontró 73 fóridos en 17 meses pertenecientes a dos especies que parasitan a $A$. robusta Borgmeier. La especie $E$. attae es registrada por primera vez atacando a $A$. colombica, por lo que la escala de hospederos que utiliza para su reproducción puede ampliarse.

Los datos obtenidos no se ajustaron al GLM, debido a que la actividad de las hormigas durante el día y las épocas del año es muy variable; esto coincidió con los resultados 
reportados por Elizalde (2009) y Gomes, Elizalde y Queiroz (2013). Posiblemente la abundancia de fóridos dependa de otras variables como las condiciones ambientales o disponibilidad de recursos (Uribe, 2013).

La mayor cantidad de $A$. colombicus en los caminos de forrajeo, concuerda con lo registrado por Feener y Moss (1990), atraídos por las cargas que transportan las hormigas y que utilizan como soporte para su ataque. Adicionalmente, también se les observó atacando a las hormigas en las bocas del nido o en la salida al basurero, cuando las hormigas transportaban cargas para ser desechadas. Este comportamiento también se refleja en especies como Apocephalus neivai, que ataca principalmente en el camino de forrajeo y en ocasiones en los basureros de hormigas cortadoras del género Acromyrmex (Elizalde, 2009). De otra parte, la abundancia de E. attae no concuerda con los resultados descritos por Eibl-Eibesfeldt (1967) y Elizalde (2009), quienes encontraron que esta especie prefiere los caminos de forrajeo para desplegar sus ataques sobre $A$. cephalotes. Esta diferencia se podría explicar si se tiene en cuenta que las áreas de desechos de esta hormiga, se ubican en el interior de los nidos, mientras que las áreas de desechos de $A$. colombica se ubican en áreas externas. De esta manera, podemos deducir que tanto la complejidad del microhábitat como la actividad de la hormiga, juegan un papel importante para el acecho y ataque por parte del fórido, generando sobre las interacciones un efecto de segregación en la abundancia y distribución de las dos especies de fóridos (Gomes et al., 2013).

Los resultados obtenidos de la presencia de A. colombicus, presente durante todo el año, difieren de lo reportado por Feener y Moss (1990), quienes encontraron que esta especie de fórido limita su presencia a la estación seca. Por el contrario, la presencia de E. attae si reflejó una mayor abundancia en dicha época seca. Las dos especies tuvieron un amplio horario de actividad en el cual atacaron a sus hospederos. Fóridos con una amplia escala temporal de actividad a lo largo del día es consistente con Bragança, Tonhasca y Della Lucia (2009) quienes dependiendo de la zona geográfica y la temporada del año, registraron para fóridos del género Neodohrniphora (filogenéticamente el más cercano a Eibesfeldtphora), actividad en los nidos de la hormiga Atta sexdens (L.) a lo largo de todo el día. Los fóridos suelen modificar sus tasas de parasitismo, dependiendo de las estaciones del año y a lo largo de diferentes generaciones (Folgarait, 2013).

Nuestras observaciones sobre el punto donde ovipone A. colombicus concuerdan con lo descrito por Fenner y Moss (1990), quienes mencionan que siempre ataca y coloca sus huevos junto al músculo abductor mandibular y cerca de la articulación clipeal de la mandíbula. Por otra parte, E. attae fue observado atacando en el gáster y el mesosoma, a diferencia de Eibl-Eibesfeldt (1967) y Elizalde (2009), quienes observaron algunos individuos de esta especie atacando a las hormigas $A$. cephalotes en la cabeza. Sin embargo, E. attae despliega un comportamiento similar al de $E$. brancai y al de E. erthali atacando a hormigas Atta bisphaerica Forel, 1908 y A. laevigata F. Smith, 1858 respectivamente, actuando mediante movimientos muy rápidos sobre la zona del gáster y ovipositan aparentemente en el ano de la hormiga (Bragança et al., 2002; Bragança, Della Lucia, \& Tonhasca, 2003). El punto de oviposición puede convertirse en una forma de segregar el recurso hospedero y la selección de puntos diferentes puede ser ventajoso para las dos especies de fóridos, pues el desarrollo de las larvas estará garantizado en caso de que juntas ovipositen una misma hormiga (Elizalde, 2009).

Diferentes estudios han mencionado la dependencia que tienen algunos fóridos de los componentes visuales para la ubicación y selección de los hospederos, como por ejemplo especies del género Neodohrniphora (Bragança et al., 2009; Gazal, Bailez, \& Viana-Bailez, 2009), por lo cual deben acercarse a distancias pequeñas o incluso entrar en contacto con las hormigas para evaluar si son hospederos ideales. De este modo, A. colombicus podría estar utilizando el tamaño y la forma de la carga como una señal visual de corto alcance, 
que evalúa para poder aterrizar sobre ellas e inspeccionar fácilmente un posible ataque. Del mismo modo, las cargas más grandes y de formas aplanadas (comprimidas bilateralmente) son más difíciles de proteger por los polizones que las cargas pequeñas (Feener \& Moss, 1990), debido a que hay una mayor área donde las moscas podrían aterrizar, razón por la cual los parasitoides como A. colombicus preferirán posarse sobre cargas grandes para poder evaluar y maniobrar su ataque.

El hecho de que E. attae y A. colombicus no discriminaran hormigas más grandes que el promedio, posiblemente se deba a que la variación del tamaño de las mismas se da en un amplio y continuo rango definido por la amplitud del ancho cefálico (Hölldobler \& Wilson 1990; Wetterer 1999), lo que dificulta una selección por parte de los parasitoides. Este resultado coincide con Elizalde (2009), que registró ataques de fóridos en mayor proporción sobre hormigas del género Atta de tamaños medianos, donde se encuentran las castas de forrajeadoras-excavadoras y que están más expuestas al ataque de fóridos, y donde solo un porcentaje menor elegía hormigas más grandes, casta de las defensoras encargadas del cuidado de la colonia y que no siempre se encuentran en las áreas de corte.

Cada parasitoide (A. colombicus y E. attae) mostró diferencias en el despliegue de sus conductas siendo específicos en aspectos como el lugar donde atacan a la hormiga y el microhábitat del nido preferido. Según Fenner y Moss (1990), quienes también describen un despliegue similar de la conducta de $A$. colombicus al atacar a $A$. cephalotes, estas diferencias pueden ser el resultado de los diversos tipos de señales que puede utilizar el parasitoide (visuales, olfativas, entre otras) para la búsqueda de sus hospederos, la resistencia que opongan las hormigas y la distribución de ambos participantes de la interacción.

Eibesfeldphora brancai y E. erthali son especies de fóridos que parasitan a las hormigas A. bisphaerica bisphaerica y A. laevigata respectivamente, exhibiendo un comportamiento similar al de E. attae, atacando rápidamente y dirigiéndose al gáster de la hormiga para ovipositar en su ano. Igualmente, Elizalde (2009) describe el comportamiento de E. attae, indicando que estos fóridos se aproximan por detrás de las hormigas y las atacan rápidamente.

Todas las conductas desplegadas por las hormigas siempre implican una interrupción del flujo normal del tráfico en la colonia. Estos comportamientos de defensa de A. colombica, muestran una similitud con los de otras especies como A. robusta ante el ataque de Neodohrniphora sp. y Myrmosicarius sp. que ataca a los fóridos o aumenta la velocidad para alejarse de ellos (Da Silva, 2011). También encontramos polizones como una respuesta de defensa ante el ataque de $A$. colombicus, en concordancia con lo registrado por Feener y Moss (1990).

La hipótesis de asimetría en las interacciones parasitoide-hospedero, indica que hay una gran variación interespecífica en los comportamientos de los fóridos, pero no en la respuesta de sus hospederos por la desventaja de las hormigas de no saber qué fórido atacará (Lapchin $\&$ Guillemaud, 2005). Si bien en nuestro estudio encontramos comportamientos defensivos de las hormigas que se solapan ante el ataque de las dos especies de fóridos, apoyando dicha hipótesis, también se encontraron ciertas respuestas diferentes ante el ataque de cada fórido que se basaron en la conducta particular de la especie de parasitoide y el microhábitat, pues los polizones y el acto de dejar caer la carga eran solo respuestas ante el ataque de A. colombicus, mientras que la captura del fórido y el ataque directo de la hormiga hacia la mosca se observó solo cuando $E$. attae era el atacante.

Ampliar el conocimiento sobre la diversidad de hospederos de una especie parasitoide (E. attae) es muy importante, pues esta información nos podría ayudar a comprender las relaciones filogenéticas que puede haber entre las especies y géneros de parasitoides con sus hospederos (Feener y Moss, 1990). Los fóridos E. attae y A. colombicus especializaron el despliegue de su conducta durante los ataques a sus hospederos, con el fin de garantizar un ataque exitoso sobre dichos hospederos, quienes 
respondieron modificando su comportamiento según el parasitoide atacante.

\section{AGRADECIMIENTOS}

Agradecimientos a la Universidad de Córdoba y a la Universidad Nacional de Colombia, sede Medellín junto con sus programas de Biología y Biociencias, por brindar las instalaciones de sus laboratorios. A Soraya Uribe Celis por su valiosa ayuda en la identificación de los fóridos y a Daniel Ochoa, por permitir el acceso al área de estudio.

\section{RESUMEN}

El éxito de las interacciones parasitoide-hospedero está determinado por la conducta de búsqueda, reconocimiento, ataque y defensa que despliegan los participantes en dicha interacción. Para comprender los patrones comportamentales entre los fóridos parasitoides y su hospedero, Atta colombica, se realizaron observaciones en un fragmento de bosque en el departamento de Córdoba, con un esfuerzo muestral de 186 horas en tres nidos de esta. Se recolectaron fóridos, hormigas atacadas y la carga que llevaban. En total se capturaron 52 individuos de Eibesfeldtphora attae y 54 de Apocephalus colombicus. Se observaron diferencias en cuanto a la forma de ataque y el microhábitat preferido por los parasitoides. No hubo preferencia aparente por el tamaño de las hormigas que atacaron los fóridos, pero seleccionaron cargas de mayor tamaño para posarse sobre ellas. Cuando las hormigas respondieron al ataque, emplearon defensas individuales (adoptando posiciones o aumentando su velocidad) o grupales (cuando hormigas vecinas atacaban al parasitoide). Los fóridos parasitoides especializan su conducta con el fin de garantizar un ataque exitoso sobre su hospedero, quien responde modificando su comportamiento según el parasitoide atacante.

Palabras clave: hormigas cortadoras de hojas, comportamiento-hospedero, comportamiento de selección, interacciones, parasitoidismo.

\section{REFERENCIAS}

Bragança, M. A., Della Lucia, T., \& Tonhasca Jr, A. (2003). First record of phorid parasitoids (Diptera: Phoridae) of the leaf-cutting ant Atta bisphaerica Forel (Hymenoptera: Formicidae). Neotropical Entomology, 32(1), 169-171.

Bragança, M. A., Tonhasca, Jr. A., \& Della Lucia, T. (2009). Biological and behavioral characteristics of
Neodohrniphora elongata Brown (Diptera, Phoridae), a parasitic fly of the leaf-cutting ant Atta sexdens rubropilosa Forel (Hymenoptera, Formicidae). Revista Brasileira de Entomologia, 53(4), 600-606.

Bragança, M. A., Tonhasca Jr, A., \& Moreira, D. (2002). Parasitism characteristics of two phorid fly species in relation to their host, the leaf-cutting ant Atta laevigata (Smith) (Hymenoptera: Formicidae). Neotropical Entomology, 31(2), 241-244.

Brown, B. V. (1997). Revision of the Apocephalus attophilus-group of ant-decapitating flies (Diptera: Phoridae). Contributions in Science, 468, 1-60.

Brown, B. V., Borkent, A., Cumming, J. M., Wood, D. M., Woodley, N. E., \& Zumbado-Arrieta, M. A. (2010). Manual of Central American Diptera (Vol. 2). Ottawa: NRC Research Press.

Da Silva Gomes, D. (2011). Ecologia de parasitóides (Diptera: Phoridae) de Atta robusta Borgmeier, 1939 (Hymenoptera: Formicidae) em ambiente de restinga (Tesis de maestría). Universidade Federal Rural do Rio de Janeiro, Brazil. Recuperada de http://www. ufrrj.br/posgrad/cpgba/teses.html

Eibl-Eibesfeldt, V. J. (1967). On the guarding of leafcutter ants by minima-workers. Naturwissenschaften, 54(13), 346-346.

Elizalde, L. (2009). Biogeografia y comunidades de fóridos parasitoides de hormigas cortadoras de hojas: diversidad del sistema y partición del recurso hospedador (Tesis doctoral inédita). Universidad Nacional de Quilmes, Bernal, Buenos Aires, Argentina.

Elizalde, L., \& Folgarait, P. J. (2010). Host diversity and environmental variables as determinants of the species richness of the parasitoids of leaf-cutting ants. Journal of Biogeography, 37(12), 2305-2316.

Elizalde, L., \& Folgarait, P. J. (2011). Biological attributes of Argentinian phorid parasitoids (Insecta: Diptera: Phoridae) of leaf-cutting ants, Acromyrmex and Atta. Journal of Natural History, 45(43-44), 2701-2723.

Feener, Jr, D. H., \& Brown, B. V. (1997). Diptera as parasitoids. Annual Review of Entomology, 42(1), 73-97.

Feener, Jr, D. H., \& Moss, K. A. (1990). Defense against parasites by hitchhikers in leaf-cutting ants: A quantitative assessment. Behavioral Ecology and Sociobiology, 26(1), 17-29.

Fellowes, M., \& Godfray, H. (2000). The evolutionary ecology of resistance to parasitoids by Drosophila. Heredity, 84(1), 1-8.

Folgarait, P. J. (2013). Leaf-cutter ant parasitoids: Current knowledge. Psyche: A Journal of Entomology, Article ID 539780, 10 pages. 
Gazal, V., Bailez, O., \& Viana-Bailez, A. M. (2009). Mechanism of host recognition in "Neodohrniphora elongate" (Brown) (Diptera: Phoridae). Animal Behaviour, 78(5), 1177-1182.

Gomes, D. S., Elizalde, L., \& Queiroz, J. M. (2013). Parasitoids of the endangered leaf-cutter ant Atta robusta Borgmeier in urban and natural areas. Revista Brasileira De Entomologia, 57(3), 335-339.

Holdridge, L. R. (1987). Ecología basada en zonas de vida. San José, Costa Rica: Instituto Interamericano de Cooperación para la Agricultura.

Hölldobler, B., \& Wilson, E. O. (1990). The ants. Harvard University Press.

Lapchin, L., \& Guillemaud, T. (2005). Asymmetry in host and parasitoid diffuse coevolution: When the red queen has to keep a finger in more than one pie. Frontiers in Zoology, 2(4), 4.

Mathis, K. A., \& Philpott, S. M. (2011). Current understanding and future prospects of host selection, acceptance, discrimination, and regulation of phorid fly parasitoids that attack ants. Psyche: A Journal of Entomology, Article ID 895424, 9 pages.

Palencia, G., Mercado, T., \& Combatt, E. (2006). Estudio agroclimático del departamento de Córdoba. Montería, Colombia, Universidad de Córdoba.
Rodríguez del Bosque, L., \& Arredondo-Bernal, H. (2007). Teoría y aplicación del control biológico. México, DF.: Sociedad Mexicana de Control Biológico.

Roitberg, B., Boivin, G., \& Vet, L. E. M. (2001). Fitness, parasitoids, and biological control: An opinion. The Canadian Entomologist, 133(03), 429-438.

Santos, A., \& Quicke, D. L. (2011). Large-scale diversity patterns of parasitoid insects. Entomological Science, 14(4), 371-382.

Uribe, S. (2013). Fóridos (Diptera: Phoridae) asociados al hábitat de hormigas cortadoras de hojas (Atta cephalotes y Acromyrmex octospinosus) y sus patrones de localización en un bosque seco tropical andino (Tesis de maestría). Universidad Nacional de Colombia, Sede Medellín. Recuperada de http://www.bdigital. unal.edu.co/9278/

Uribe, S., Brown, B. V., Bragança, M. A., Queiroz, J. M., \& Nogueira, C. A. (2014). New species of Eibesfeldtphora Disney (Diptera: Phoridae) and a new key to the genus. Zootaxa, 3814(3), 443-450.

Wetterer, J. K. (1999). Ecology and evolution of worker size-distribution in leaf-cutting ants (Atta spp. and Acromyrmex spp.). Sociobiology, 34, 119-144. 
